\title{
La disputa entre China e India y la redefinición de la geopolítica asiática
}

\author{
The China-India dispute and the redefinition of Asian geopolitics
}

DOI: https://doi.org/10.22456/2178-8839.107055

Andres Ernesto Ferrari Haines Universidade Feral do Rio Grande do Sul, Porto Alegre, Brasil afhaines@hotmail.com

Erik Herejk Ribeiro Instituto Sul-Americano de Política e Estratégia, Porto Alegre, Brasil erik_ribeiro@yahoo.com.br

\section{Resumen}

El enfrentamiento chino e indio en junio de 2020 a lo largo de su disputada frontera, pese ser un tema sin resolver desde hace tiempo, ha adquirido un nuevo significado en las actuales tensiones geopolíticas globales. Podría estallar en una profunda disputa que involucrará a los dos gigantes asiáticos en una confrontación mucho más amplia. Por su relevancia conjunta, el conflicto afectaría no solo a países cercanos, sino a todo el mundo, en medio de la confrontación sino-estadounidense. Este artículo analiza los diferentes aspectos que están afectando este malentendido, vinculando preocupaciones históricas con desarrollos recientes. En base al análisis geopolítico clásico del Rimland, se destacan las diversas limitaciones e impulsos que definirán el futuro de los dos países que ahora se encuentran entre las mayores economías mundiales. El análisis apunta a las ansiedades que llevaron al choque fronterizo y a las inseguridades mutuas con respecto al comportamiento de cada uno, particularmente en sus relaciones con terceros países. Frente a esto, una solución de la disputa parece fuera de alcance, ya que se ha convertido en parte del cambio geopolítico del presente siglo, que una vez más está poniendo a Asia en el centro de la competencia mundial.

Palabras claves: Relaciones sino-indias; Geopolítica; Asia; China; India;

\section{Abstract}

The Chinese and Indian clash in June 2020 along their disputed border, despite being a long non-resolved issue, has acquired new meaning in the present global geopolitical tensions. It could erupt into a deep feud which will involve the two Asian giants in a much wider confrontation. Because their relevance in world affairs, this conflict would affect countries not only nearby, but all over the globe, amidst the substratum of the ongoing confrontation China is having with the United States. This article analyses the different aspects that are affecting this misunderstanding, linking historical concerns with recent developments. Based on the classical geopolitical analysis of the Rimland, it envisions the various constraints and impulses that will define the future of the two most populous countries of the Eurasian landmass, which are now among the largest global economies. The analysis points to the anxieties that drove China and India to the frontier clash and the mutual insecurities with regard to each other's behavior, particularly in their relationships with third countries. In lieu of this, a solution of the dispute seems out of reach as it has become part of the present century's geopolitical shift which is once again putting Asia in the center of the global competition.
\end{abstract}

Keywords: Sino-Indian relations; Geopolitics; Asia; China; India. 


\section{Introducción}

El ascenso relativo de Asia en el siglo XXI ha trasladado gradualmente el eje geopolítico global del Atlántico Norte y del Mediterráneo hacia los océanos Índico y Pacífico. En consecuencia, las disputas históricas entre China e India, las dos principales civilizaciones y potencias asiáticas, adquieren un nuevo significado, que sobrepasa sus fronteras. El objetivo de este artículo es situar la rivalidad sino-indiana como uno de los aspectos centrales en la redefinición del orden asiático y, por ende, de la geopolítica global. Su hipótesis es que el conflicto de frontera entre China e India simboliza una disputa mayor entre dos pívots geopolíticos del siglo XXI.

La dinámica de fondo para los cambios geopolíticos en Asia puede entenderse mejor a través de la inclusión de la geopolítica clásica en las lecturas contemporáneas de relaciones internacionales. Se utiliza aquí la proposición del do Rimland para analizar porque los conflictos entre China e India se convertirán en elementos claves en este proceso. El Rimland es definido como las regiones densamente pobladas en los extremos geográficos de Eurasia: oeste (Europa), sur (subcontinente indiano) el este (Asia Oriental).

La teoría de Nicholas Spykman sobre el Rimland ayuda a comprender el ascenso asiático como nuevo centro dinámico del Sistema Internacional. Spykman (1944) propone, en forma contraria a Halford Mackinder y Alfred Mahan, que nunca hubo una oposición simple entre potencias terrestres versus potencias navales. Los grandes países “anfibios” del Rimland serían los verdaderos pívots de la historia, pues son grandes matrices civilizatorias y tiene el desafío geográfico de estar en conflicto constante atrito con potencias terrestres e insulares. Por otro lado, los países do Rimland son los únicos (además de Estados Unidos) con suficiente capacidad de tener influencia y proyectar poder simultáneamente en franjas territoriales y marítimas.

El caso de Spykman y la teoría del Rimland ilustran el potencial de China e India. Las dos sociedades tienen en común una historia de gran civilización que determinó la formación política de Asia y poblaciones contemporán eas de más de mil millones de personas. También se destacan porque los dos serían capaces de dominar una integración de la masa Eurasiana, así como los flujos marítimos de los océanos Índico y Pacífico, que responden por gran parte del comercio mundial. De este modo, el retorno de Asia como centro del sistema debe ser caracterizados por un patrón de cooperación y conflicto entre sus dos principales exponentes (RIBEIRO, 2019, p. 51-52).

Tres vectores claves entran en juego en esta dinámica. En primer lugar, la disputa fronteriza entre China e India es cada vez menos un asunto estrictamente bilateral. Los impactos del conflicto sino-indio repercuten en el Sur de Asia, dado que India ha perdido parte de su tradicional influencia regional para China y en el Pacífico Occidental, y debido a las tensas disputas marítimas de China con sus vecinos. En segundo lugar, la rápida modernización de China y el mayor poder que obtuvo en relación a India generaron una interdependencia asimétrica a favor de Pequín. Estas diferencias acentúan las contradicciones económicas indias y la disputa respecto a las diferentes propuestas de integración regional en Asia. Por último, el triángulo estratégico entre China, India y Estados Unidos se ha desequilibrado en favor del eje Nueva DelhiWashington. Mientras los indios desean preservar su autonomía estratégica, la expansión del poder material y de la influencia china acelera una alianza llena de incertidumbre a largo plazo.

La coyuntura actual exhibe crisis diversas, desde la guerra comercial entre China y Estados Unidos a los nuevos conflictos en la frontera sino-india y el inicio de boicots económicos de India a productos e inversiones chinas. En medio de la pandemia mundial del COVID-19 se produjo un violento choque entre estos dos conspicuos representantes del Sur global. El 15 de junio de 2020 un enfrentamiento entre ambos fue el más grave desde 1975, la última vez que un conflicto en la frontera entre China e India terminó con muertos. Pero si bien la causa fue una disputa fronteriza nunca resuelta, que ya había generado varias escaramuzas, en esta ocasión aparenta expresar profundos cambios en la geopolítica global, en la cual tanto uno como el otro han adquirido una relevancia mundial más cercana a la que tuvieron en tiempos previos a ser objeto de la colonización occidental. 
Es más, al exteriorizar tensiones en rivalidades actuales por el poder global, el choque expone el movimiento en curso de (re)ascenso de Asia y del relativo declive de los poderes occidentales. El conflicto está revelando una fuerza centrípeta involucrando otras naciones, dentro y fuera de la región. Esto significa que su desenlace es parte de la redefinición del orden regional asiático y, por el peso mundial de China e India, del reajuste internacional que se está desarrollando. De esta manera, cómo los dos conduzcan este conflicto puede llevar a una reformulación de los conceptos de Norte-Sur y, por ende, del entendimiento o visión del Sur Global.

El artículo se divide en cuatro secciones adicionales. La segunda sección explica los orígenes de la disputa fronteriza y relaciona el deterioro reciente de las relaciones sino-indianas al conflicto territorial. La tercera sección analiza como el aumento de estas tensiones se refleja en el debate de la actual coyuntura, trayendo temas de soberanía territorial, económica y tecnológica al centro de la agenda bilateral. La cuarta sección sitúa la competencia sino-india en la nueva geopolítica asiática. Se argumenta que la dinámica de percepción de cerco entre los dos país es se ha acentuado: ya sea en relación a la influencia china en el subcontinente indiano y en el Océano Índico o a las asociaciones de India con Estados Unidos y países del este Asiático. Dos símbolos recientes de esta competencia son el Belt and Road Initiative (BRI) del lado chino y la asociación del Cuadrilátero Marítimo (Quad) del lado indio. En la última sección, se presentan las principales conclusiones del artículo y una breve perspectiva sobre el futuro de las relaciones entre China e India en el contexto geopolítico de Asia.

\section{El origen del conflicto}

La disputa entre China e India remonta a una demarcación fronteriza no resuelta desde el origen de la formación moderna de ambas. Tanto India, que nace de la división territorial de la antigua India colonial británica en 1947, como China, que surge de la Revolución Comunista de 1949 - que es origen reconocido de su realidad actual -debieron lidiar con grandes desafíos que les generaron inseguridad. Si bien la simultánea disparada de la Guerra Fría tuvo un impacto directo más en China que en India, la división de la India colonial le daría en Pakistán un rival de enfrentamiento constante. En particular, porque, habiendo surgido con menos recursos que la India, Pakistán procuró compensar esto entrando en la disputa EE.UU.-URSS. Así, bastante a su pesar, la dinámica externa india reiteradas veces debió abandonar tácticamente su estrategia de país no-alineado - movimiento del cual fue gestor - e inmiscuirse en alianzas externas.

Por otro lado, China e India, pese a su larga historia, tuvieron que lidiar con los desafíos de naciones recién creadas y con un desempeño económico que sería bastante pobre que debía satisfacer las mayores poblaciones en el mundo. Estas inseguridades experimentadas, sumadas a la percepción de fragilidades propias, condujeron a que se viesen mutuamente con desconfianza. En particular, la percepción china de una amenaza en el Tíbet fue fundamental para desencadenar un espiral de acciones y reacciones en la frontera (GARVER, 2006). Así, la disputa fronteriza nunca definida, en ese marco, acabó en la breve guerra en 1962 ganada por China.

La guerra trajo cambios permanentes en la dinámica sino-india de cooperación y conflicto. Desde 1962 hasta 1988, las relaciones estuvieron prácticamente congeladas. Luego de la guerra, ambos dejaron el asunto sin una solución definitiva volviéndose un factor de fricciones recurrentes de distinta intensidad. No obstante, la escalada de 1975 es sintomática por constituir la última de gravedad hasta la de este año. Además, porque en los 70 ambos pasaron a convivir en un marco geopolítico nuevo que iría a viabilizar trayectorias de grandes transformaciones para ambos. Luego del encuentro entre Nixon y Mao, China se aproximó a EE.UU. para superar las inseguridades que le provocaba la vecina Unión Soviética. Al mismo tiempo, India pasaría a sentirse más fortalecida luego que su tercera guerra contra Pakistán en 1971 lo desmembraría con la creación de Bangladesh (SINGH, 2019; MADAN, 2020b; SAUTER, 2020).

Sin embargo, las relaciones chino-indias adquirieron un nuevo carácter a finales del siglo XX. Las tensiones en la frontera fueron dejadas de lado por nuevos líderes en Nueva Delhi y Pequín, que se volcaron a sus respectivas agendas 
de desarrollo como prioridad estratégica. Así, luego de un grave enfrentamiento fronterizo en 1986-87, en un encuentro histórico, el Primer Ministro indio Rajiv Gandhi viajó en 1988 para acordar una solución temporaria a sus disputas con el líder chino Deng Xiaoping (FANG, 2013).

En 1998, hubo otro cambio estructural: India se convirtió en potencia nuclear, desafiando el orden internacional liderado por las potencias P-5 (China, Estados Unidos, Rusia, Inglaterra y Francia) y colocándose como candidata de potencia de primer rango. Más que eso, sus experimentos nucleares fueron una respuesta directa de sus elites a la percepción de que a China estaba incentivando la nuclearización de Pakistán como forma de contener a India (FANG, 2013).

La rivalidad sino-indiana pareció, durante bastante tiempo, como una disputa donde uno sólo de los contrincantes estaba en el ring. Históricamente, la República Popular de China tuvo mayores preocupaciones en otras frentes, como las recurrentes crisis en Corea, en Vietnam y en el estrecho de Taiwán, además de una Unión Soviética hostil a partir de la década de 1960. Así, la percepción de amenaza asimétrica entre China e India es interpretada como uno dos grandes escollos a la estabilidad en las relaciones bilaterales: los indios perciben cada acto chino de forma mucho más consecuente que del otro lado (FANG, 2013).

No obstante, esta dinámica ha manifestado cambios a partir de la perspectiva de que India debe convertirse en breve la segunda mayor potencia asiática y un polo de poder del Sistema Internacional (RIBEIRO, 2019). Aun así, las elites estratégicas chinas sólo perciben a India como amenaza en un escenario de asociación con Estados Unidos y otras potencias regionales como Japón. En menor medida, las relaciones históricas de India con líderes tibetanos también es motivo de preocupación en Beijing (FANG, 2013). China, que siempre ha visto a India como una potencia regional de menor importancia a escala mundial o como amenaza (SHIRK, 2004), ha experimentado un dilema más agudo: Por un lado, hay una creciente rivalidad por la influencia en Asia; por otro, existen objetivos comunes de desarrollo económico y reforma del orden internacional para acomodar los intereses de las potencias emergentes en el Sur Global (TELLIS; MIRSKI, 2013).

En el último medio siglo, ambos se ubicaron en las principales economías del mundo. Una consecuencia de esto es que sintieron la necesidad de aproximarse debido a que no estaban contemplados en las estructuras de gobernanza internacional existentes. Así, uniéndose a Brasil, Rusia, y Sudáfrica en la agrupación BRICS y en otras instituciones regionales, organizaciones bancarias y multilaterales (ej. Banco de Inversión Asiática en Infraestructura), intentaron obtener mayor espacio en la configuración del orden global. Al mismo tiempo, incrementaron sus vínculos económicos, destacándose que China pasó a ser una gran fuente de inversiones para India y el mayor socio comercial indio entre 2013 y 2019, cuando pasó a ser Estados Unidos.

Pero recientemente los caminos volvieron a bifurcarse, y la cuestión fronteriza no resuelta pasó a evidenciar ese desencuentro. Hubo enfrentamientos en Depsang en 2013, en Chumar en 2014 y, sobre todo, en Doklam en 2017 . Este último se debió a que India se opuso a la extensión de una carretera por parte de China en la meseta de Doklam, donde los dos países se encuentran con Bután. Ese territorio bajo control chino también es reclamado por el aliado de la India, Bután. La Cumbre de Wuhan a principios de 2018 volvió a bajar las tensiones y en octubre de 2019 el presidente chino Xi Jinping y el primer ministro indio Narendra Modi se reunieron procurando llevar las relaciones a mayores alturas en 2020 afirmando conmemorar el $70^{\circ}$ aniversario de sus lazos formales mediante 70 actividades conjuntas.

El choque en junio último ocurrió en varios lugares del sector occidental de su frontera disputada (la zona conocida como Aksai Chin), donde hay cerca de 38.000 kilómetros cuadrados bajo control chino. Pero las tensiones que llevaron a él surgieron desde abril en la medida que China trasladaba un gran número de tropas a la Línea de Control Actual (LAC), la frontera de hecho que separa a las fuerzas chinas e Indias, en tres áreas del sector occidental. Por otro lado, India

\footnotetext{
${ }^{1}$ Según Madan (2020a, n.p): “En cada caso, la India acusó a China de intentar cambiar unilateralmente el status quo territorial avanzando tropas y estableciendo una presencia permanente en posiciones que no debían ocupar. Otros problemas no resueltos siguen atormentando la relación bilateral, incluida la presencia del Dalai Lama y los refugiados tibetanos en la India (que exaspera a China), el control de China de las aguas del río Brahmaputra (una fuente de preocupación para la India), y lo que Nueva Delhi ve como una relación económica desequilibrada".
} 
viene construyendo una carretera en el valle del río Galwan, una zona delimitada por Ladakh y Aksai Chin, controlado por la India por un lado y administrado por China por el otro.

De hecho, China viene desde hace décadas construyendo carreteras a lo largo de la frontera con cerca de 3.500 km (2.200 millas). India también está ampliando accesos a lo largo de línea fronteriza occidental, incluyendo ramas a rincones remotos de la frontera. A lo largo de la frontera en disputa durante la última década, la India ha construido infraestructura, incluidas carreteras y puentes para tratar de igualar similares esfuerzos chinos. Esa dinámica marcada por la construcción tanto de uno como de otro de carreteras en torno a la frontera fue desencadenando la confrontación reciente bajo un marco en que los dos veían los movimientos del otro como amenazas (GUPTA, 2014).

De esta manera, en los últimos tiempos ambos han estado marcando mayor presencia en torno a esa región fronteriza que no posee una delimitación clara que sirvió para provocar un enfrentamiento fronterizo que no se agota en él, sino que constituye la expresión de un desentendimiento con razones muchas más profundas. Es por eso que la reacción de la India tras el choque fue acusar a China de haber roto los acuerdos de paz y tranquilidad de 1993 y de los parámetros políticos y principios para la resolución de la cuestión de frontera de 2005.

La declaración india pareció casi un ultimátum amenazando con que todos los avances logrados en la relación bilateral en las últimas tres décadas se perderían si China no desescalaba y desactivaba su presencia militar en la frontera. Así, el ministro de relaciones exteriores indio Subrahmanyam Jaishankar le dijo a su homólogo Wang Yi que "este desarrollo sin precedentes tendrá un impacto grave en la relación bilateral" (MEA, 2020). Sin embargo, no resulta claro cuál camino le convendría seguir a India, ni tampoco si para China le es lo más conveniente convivir con una India en disyuntiva en medio del contexto de su disputa con Estados Unidos.

\section{Um conflito que ninguno quiere}

De esta forma, el choque de junio sobrepasa un desentendimiento fronterizo y expresa ansiedades mutuas en relación a sus movimientos de política externa. Curiosamente, ambos se perciben como siendo cercados por el otro. India por China en su construcción de la ruta de la seda moderna, que la lleva a un acercamiento a su clásico rival Pakistán, además de Bangladesh, Nepal y Sri Lanka; y en la región más amplia del Océano Indico, en una proyección que parece buscar catapultarla en la hegemonía regional. Esta percepción está simbolizada por la teoría del Collar de Perlas, según la cual los chinos estarían construyendo una red de bases navales en la vecindad india para contener su expansión marítima (BREWSTER, 2017).

China, por su parte, se siente cercada por India, dada su participación en distintas agrupaciones con Estados Unidos, Australia, Japón y algunos países del sudeste asiático. Como afirma Madan (2020a), India cree que China busca obstaculizar sus ambiciones en la escena internacional bloqueando su pertenencia a organizaciones como el Grupo de Proveedores Nucleares y el Consejo de Seguridad de las Naciones Unidas. Mientras China siente que India amenaza sus intereses en la medida en que apoya el accionar de EE.UU. en instituciones multilaterales como el Grupo de Acción Financiera, las Naciones Unidas y la Organización Mundial de la Propiedad Intelectual.

Zhang Jiadong, de la Universidad Fudan en Shanghai, sostiene que antes los dos países no otorgaban mucha importancia a los lazos bilaterales, pero ahora la relación no solo adquirió gran importancia para ambos porque tienen mucha interdependencia en muchos frentes, sino porque tienen, cada uno, relaciones con otros países importantes que no han aclarado en qué consiste su posicionamiento (JIADONG, 2020).

Posteriormente apunta que, para mejorar la relación bilateral, deben establecer nuevas normas de interacción porque el reciente choque constituyó “una manifestación de tensiones estratégicas” porque cuando eran más débiles tenían menos posibilidades de encontrarse en la frontera o en alta mar, pero ahora, que son más poderosos, están aumentando las actividades en estas áreas con mayores riesgos (JIADONG, 2020). Asimismo, observa que en el presente 
siglo mientras China viene aumentando su influencia en Asia oriental, India lo está haciendo en el sur de Asia, y que, adicionalmente, este aumento de la fortaleza nacional "también ha traído nuevos requisitos para los dos países en términos de relaciones bilaterales y globales”. Además del desarrollado rápido y los cambios internacionales, Jiadong observa que también han intensificado su sentido del honor nacional, haciendo que sus representantes "se enfrenten tanto a mayores expectativas como a una mayor presión” (JIADONG, 2020, n.p.).

Académicos como Fravel (2020) afirman que China manifiesta una “obsesión” por su soberanía que se expresa en una necesidad de proyectar fuerza. Así explica el choque fronterizo con la India, en especial ante el riesgo de provocar una aproximación entre India y Estados Unidos. Para él, en ese caso, así como en Hong Kong, Taiwán y en los mares de China Oriental y Meridional, “China quiere transmitir fuerza”. Fravel argumenta que China ha adoptado un enfoque más estridente de soberanía desde que Xi Jinping se convirtió en el secretario general del Partido Comunista Chino (PCC). La relación se vincula al logro del "sueño de China" ${ }^{2}$, que ha llevado a China a recuperar territorios relevantes para la soberanía nacional. Esas acciones son representadas por: "[la captura de] grandes extensiones de tierra en lo alto de arrecifes para construir bases militares en el Mar de China Meridional, [...] patrullas de sus buques de guardacostas dentro de las aguas territoriales que disputa con Japón en el Mar de China Oriental”, además de sus actuaciones en relación a Hong Kong y Taiwán (FRAVEL, 2020).

Chaudhuri (2020) señala que Xi expresó varias veces la importancia que India tenía para China. Comenta que, según el embajador indio Ashok Kantha, Xi le dijo que veía la mejora de los lazos como una "misión histórica" y que veía a ambos países trabajando en cuestiones globales. Kantha también afirmó que "Xi se hizo cargo personalmente de la política de la India. Cuando el ministro de Relaciones Exteriores visitó la India, se le dio el título adicional del emisario especial de Xi" (CHAUDHURI, 2020, n.p.). Kantha sostiene que Xi también solicitó ser primer líder extranjero en reunirse Narendra Modi luego de ser elegido. India considera que "la evidencia de la retórica de Xi que se tradujo en acción sobre el terreno era escasa” (CHAUDHURI, 2020, n.p.).

Siendo ese el entendimiento en la India, más allá de cuál era el estado de las relaciones antes del choque en Galwan, lo concreto es que luego del enfrentamiento, India manifiesta ya no creer que Beijing busque genuinamente un "nuevo paradigma" en el vínculo. Para Madan (2020a), el combate muestra cómo China está perdiendo la India y que por eso el episodio tiene el potencial de marcar lo que algunos analistas han llamado "un momento decisivo" en la relación entre China e India. La evaluación considerada en Nueva Delhi, afirma Madan (2020a), es que China ha "perdido" a la India debido a sus acciones impulsivas en el valle de Galwan y “que las ganancias tácticas a corto plazo a lo largo de la Línea de Control Actual afectarán negativamente el panorama general para la República Popular en su centenario en 2049”. De acuerdo al especialista en asuntos de defensa Bhaskar, la visión de China que comienza a surgir en su país es "la perspectiva de un matón solitario y hosco que busca imponer la hegemonía china a una Asia que resistirá ese expansionismo” (BHASKAR, 2020, n.p.).

India, así, se ha endurecido contra China y la herramienta que encontró más a mano para hacerlo es buscar golpearla económicamente. Las agencias de seguridad indias han vedado a empresas que se creía que poseían vínculos con el gobierno chino en sectores críticos, en particular telecomunicaciones, educación superior y suministro y distribución de energía. El comunicado del Ministerio de Finanzas de la India sostuvo que había modificado sus Reglas Financieras Generales "para permitir la imposición de restricciones a los licitadores de países que comparten una frontera terrestre con la India”. El Ministerio justificó su acción por "motivos de defensa de la India, o asuntos relacionados directa o indirectamente con la seguridad nacional" (MF, 2020, n.p.). La afirmación se entendió largamente como estando dirigido en forma clara a China (PTI, 2020b).

\footnotetext{
${ }^{2}$ El autor basa su visión en las declaraciones de Xi: "ningún país extranjero debería esperar que negociemos nuestros intereses fundamentales" o esperar que China "se tragara el amargo fruto" de las invasiones de sobre su "soberanía". También cita a Xi en 2018 afirmándole al Secretario de Defensa de Estados Unidos James Mattis que China "no puede perder ni un centímetro del territorio dejado por nuestros antepasados" (FRAVEL, 2020, n.p).
} 
Al mismo tiempo, Narendra Modi apareció en la televisión nacional y pidió a sus conciudadanos que reduzcan la dependencia de la India de las importaciones y se conviertan en AatmaNirbhar, el término hindi para autosuficientes. Se renovaron una serie de campañas de boicot a China en todo el país, mientras que las plataformas de medios de comunicación indias se dedicaron a atacar pesadamente al dragón. La Confederación de Todos los Comerciantes de la India (CAIT) sostuvo que movilizará a boicotear artículos chinos y apoyar los productos propios, publicando una lista de 500 categorías de artículos importados de China que podrían intercambiarse por productos de la india, como prendas de vestir, electrónica de consumo y juguetes (PTI, 2020a).

Sin embargo, India necesita de inversiones externas. Apenas en 2019, ingresaron US\$ 49 mil millones en inversión extranjera directa para crear puestos de trabajo, apoyar el desarrollo de infraestructura e introducir nuevas tecnologías. Este asunto fue tratado por el medio alemán Deutsche Welle (2020) en que indaga la factibilidad que la economía india pueda prescindir de China. La publicación cita a Biswajit Dhar, de la Universidad Jawaharlal Nehru de Nueva Delhi, que sostiene que India es un país con recursos limitados y está buscando que ingrese bastante inversión extranjera, por lo que alerta que el boicot podrá dañar la economía india. Además, Dhar destaca la importante influencia comercial de China con un déficit en torno a 65.000 millones de dólares, lo que puede perjudicar el desarrollo en largo plazo porque gran parte de las compras que le hace India consiste en bienes de capital y otros insumos necesarios para la producción doméstica.

Por otro lado, el especialista en tecnología Sahil Bhalla adicionalmente destaca la importancia que tienen los aplicativos chinos en la economía india (DW, 2020). En consecuencia, afirma que para India será complicado deshacerse de los productos chinos "de la noche a la mañana". Para que eso sea factible, continúa, cualquier campaña que consista en el rechazo de productos chinos sólo puede ser efectiva si India tiene "alternativas lo suficientemente buenas". Es escéptico que India pueda reemplazar todos los productos chinos teniendo en cuenta que las empresas chinas han inyectado miles de millones en la escena tecnológica de la India y han contratado a muchos empleados locales.

La dinámica en que se vienen encontrando China e India fue alarmantemente sintetizada por Orchard en 2018:

Siendo realistas, ninguno de los dos países tiene mucho interés en jugársela por obtener la supremacía en el IndoPacífico. Sin embargo, como lo ilustran el sentido de urgencia con que India ha estado tratando de evitar una confrontación importante, fuerzas subyacentes de todos modos están empujando a los dos lados hacia un ciclo auto perpetuo de competencia de suma cero. Y cuánto más profundamente China e India se hundan en esta espiral, más difícil será para cualquiera de los dos retirarse (ORCHARD, 2018a , n.p.)

\section{En el epicentro del futuro geopolítico asiático y global}

A nivel mundial, India posee la séptima área geográfica, la segunda población y la quinta economía. Su transformación en las últimas décadas ha sido impresionante, pero queda bastante detrás de la realizada por China - que de economía similar pasó a ser entre cinco y seis veces mayor. Juntos China e India son vectores cruciales para explicar por qué “el centro de gravedad global, tanto militar como económicamente, está cambiando de Oriente Medio al Indo-Pacífico” (FEDIRKA, 2020). Estimativas recientes señalan que el PBI asiático puede superar la producción sumada del resto del mundo. En 2030, se espera que el PBI asiático aumente hasta representar 60\% del PBI mundial (YENDAMURI; INGIZILIAN, 2019).

Pero en esa dinámica, la proyección china devela las muchas restricciones que India enfrenta. Tradicionalmente, las cuestiones internas, la preocupación con Pakistán y el acompañamiento de lo que hacía China, concentraban la atención de India. Actualmente, la fortaleza militar china se desequilibró fuertemente contra India, en un marco en que Pakistán se convirtió en aliado central del proyecto chino de construir una red de infraestructura en Asia central bajo su Belt \& Road Initiative (BRI). 
Frente a este desequilibrio, Modi ha establecido la meta de llegar a un PBI de 5 billones de dólares para 2024, lo que convertiría a la India en la tercera economía más grande del mundo, En vista de esto, en los últimos seis años, Modi avanzó en reformas económicas encaminadas a desarrollar una economía nacional más coordinada y robusta ${ }^{3}$. Al mismo tiempo, el rápido crecimiento que la economía india ha tenido en las últimas décadas la ha obligado a relacionarse externamente en un grado mayor que antes. Una preocupación especial es que su floreciente economía depende de energía extranjera por lo que debe asegurar el acceso a más suministros y desarrollar la infraestructura de seguridad para protegerlos.

Para avanzar en este propósito, Modi intenta obtener apoyo internamente para construir esa base económica más sólida, promoviendo el nacionalismo hindú y reuniendo a la mayoría de la población bajo una sola bandera. Sin embargo, este proyecto encuentra dificultades de implementación en base a la estructura de la sociedad india en la cual el hinduismo es también una religión, $\mathrm{y}$, por esa causa, ha sido motivo de enfrentamientos con otros grupos religiosos minoritarios. Especialmente, ha sucedido con los musulmanes que conforman el 14 por ciento de la población india y a menudo son blancos de violencia religiosa y social ${ }^{4}$. El sustrato de esta cuestión se encuentra en el hecho de que la población del país se encuentra muy segmentada por idioma, religión y un complicado sistema de castas. Sus Estados y territorios gozan de un alto grado de autonomía y tienen sus propios sistemas regulatorios que operan paralelamente al gobierno central. Es esta estructura social que, precisamente, está llevando a Modi procurar establecer un gobierno central más fuerte para hacer frente a los inéditos desafíos externos que la India está afrontando 5 (FEDIRKA, 2020).

En el caso concreto de China, la percepción de la India es sentirse rodeada por sus movimientos a medida que se expande a la cuenca del Océano Índico y se relaciona con países del sur de Asia. Históricamente, estos países se encuentran firmemente dentro de la esfera que India entiende es su espacio de influencia tradicional - desde Sri Lanka hasta Nepal y las Maldivas. En gran medida, la estrategia china para esta proyección es su mayor capacidad económica, expresada por medio de constituir una red regional de inversiones de infraestructura de BRI. En base a esto, Beijing pasó a adquirir derechos para construir puertos de aguas profundas estratégicamente ubicados, entre otros, en todo lo que India considera es su periferia. Según Hindustan Times (2020), India considera que China tratará inevitablemente de entrar al Océano Índico en su búsqueda de convertirse en potencia global, del mismo modo que ha reclamado grandes porciones del disputado Mar de China Meridional.

India, en respuesta, se ha acercado a Indonesia, Maldivas, Mauricio, Seychelles, Sri Lanka, Vietnam y Madagascar, para evitar que China expandiera su presencia. También informa que fuentes oficiales de la marina india consideran que China busca abrir múltiples rutas hacia el Océano Indico para superar el dilema de Malaca ${ }^{6}$ (HT, 2020).

\footnotetext{
${ }^{3}$ Fedirka (2020, n.p) así resume las iniciativas económicas recientes del gobierno indio: "Modi introdujo un esquema de desmonetización y modernizó el sistema tributario sobre bienes y servicios. Hizo varios cambios regulatorios para tratar de fomentar la inversión extranjera en áreas previamente dominadas por el estado o, con la ayuda del estado, pequeñas empresas locales. El gobierno todavía está trabajando para limpiar el sistema financiero del país, reformar los procedimientos de quiebra y aflojar los controles de precios en un esfuerzo por crear un entorno más favorable para las empresas. También ha apoyado el desarrollo de 25 sectores, como la fabricación para ladefensa, los productos farmacéuticos, los textiles y los componentes del automóvil - cuando la India tiene una ventaja comparativa para atraer inversiones y promover las exportaciones. La reciente crisis desencadenada por la pandemia sirvió como una oportunidad más para que Modi impulsara reformas en la agricultura, el carbón y la privatización como parte de los estímulos y el paquete de recuperación. A nivel macro, hay señales de que los movimientos han tenido un cierto éxito. Desde 2014, la economía de la India ha pasado de ser la décima a serla quinta más grande del mundo. La inversión extranjera directa ha aumentado de \$190 mil millones en $2009-14$ a \$284 mil millones en 2014-19”.

${ }^{4}$ Fedirka (2020, n.p) apunta que: "El año pasado, el gobierno aprobó el proyecto de ley de enmienda de ciudadanía, que excluía a los musulmanes de los grupos de inmigrantes ilegales de Bangladesh, Afganistán y Pakistán elegibles para la ciudadanía en la India. Según la ley, los musulmanes que huyen de estos países no son elegibles para la ciudadanía porque no se consideran grupos minoritarios en sus países de origen. También hay un impulso para implementar un registro ciudadano nacional para 2024. El registro requeriría que las personas que viven en la India probaran su ciudadanía india. Si no pueden, serían considerados no ciudadanos y se enfrentarían a derivaciones legales como la deportación y la denegación de servicios. Tanto el proyecto de ley de enmienda de ciudadanía como los planes para un registro nacional han sido muy controvertidos y han producido una fuerte reacción política contra el gobierno".

${ }^{5}$ En las palabras de Fedirka (2020, n.p): "Durante gran parte de su existencia post-independencia, la India ha tenido un sistema federal en el que los estados y los territorios sindicales han sido bastante autónomos. Esto dio lugar a un gobierno nacional más débil y un desarrollo desigual, pero era un precio que valía la pena pagar porque la descentralización fue vista como la mejor manera de evitar que los estados se separaran y mantener a la India intacta, lo que, después de la partición, no era una pequeña hazaña. Sin embargo, para poder aumentar su poder en el extranjero, se necesitaba un gobierno central más fuerte, y esto requería una población unida y un mensaje unificador".

${ }^{6}$ El Almirante Arun Prakash, ex jefe de la marina india, afirma que "es una realidad” que China se desplegará navalmente en el Océano Indico una vez que su poder "cruce un cierto umbral", mientras otro oficial indio sosteniendo que China está reclamando casi el 90\% del Mar de China Meridional, por lo que India debe evitar “que ese escenario se desarrolle en el Océano Indico”(HT, 2020, n.p).
} 
Este dilema constituye lo que consideran es la debilidad estratégica de China, porque precisa tener relaciones amistosas con las potencias navales que controlan el estrecho de Malaca (Singapur, Malasia e Indonesia), para evitar sufrir interdicciones en líneas de suministro que le son vitales. Un volumen significativo (más del 80\%) de las importaciones de petróleo de China pasan a través de ese estrecho que conecta el Océano Indico y el Mar de China Meridional. En consecuencia, India está tratando urgentemente de expandir su propia presencia naval en esa región ${ }^{7}$. Desde las costas del sudeste de África hasta la boca del estrecho de Malaca y, cada vez más, hacia el este y el sudeste de Asia, está intentando asociarse con los países de esa zona económica y militarmente.

Este comportamiento indicaría que India estaría dejando atrás su tradicional postura externa de país noalineado. Señales en ese sentido se observaron en 2019 con las palabras de Subrahmanyam Jaishankar, cuando sostuvo que “no-alineados” hacía referencia a "una época y un marco geopolítico particular", dando a entender que la situación actual sería distinta. De esta manera, si bien mantuvo la visión clásica de la India de no integrar algún tipo de alianza, no obstante, expresó que los cambios globales en curso estaban abriendo espacios para potencias medias como era la India (RAJA MOHAN, 2019). Sin embargo, Lin Minwang, del Centro de Estudios de Asia del Sur de la Universidad de Fudan, en China, en entrevista a Global Times (2020), da a entender que en cuestiones de seguridad y defensa en la región indo-pacífica, India se había asociado con Estados Unidos, aunque evite definirse abiertamente.

El comentario del medio chino expresa las connotaciones para ella del comportamiento chino. Esto porque la opción para India de aliarse con otra potencia se lee en buscar equilibrarse frente a China. Y, concretamente, hacerlo la acerca a EE.UU. quien viene cortejándola en su disputa con China, porque India es su candidato ideal para contraponer a China en Asia por su peso económico, político y militar ${ }^{8}$.

Así, si se le presenta a India la oportunidad de tomar un papel proactivo en la conformación de los asuntos internacionales para adaptarlos a sus intereses. Al mismo tiempo, India, en principio, continúa procurando evitar que se forme un sistema de alianzas fijas como sucedió durante la Guerra Fría. Por ese motivo, el gobierno indio continúa apostando a la Asociación de las Naciones del Sudeste Asiático (ASEAN) como punto de equilibrio en la búsqueda por la multipolaridad regional (MEA, 2018).

Económicamente, el acercamiento con EE.UU. también significa que India se está colocando como alternativa para las empresas estadounidenses y japonesas que buscan retirarse de China, lo que, es claro, tensionará más las disputas sino-indias. Así, para la próxima década será determinante de la reorganización de las cadenas productivas en Asia a partir de los efectos de la guerra comercial China-EUA. Además de la India, otros países del Sudeste Asiático como Vietnam, Malasia, Tailandia e Indonesia compiten fuertemente dentro de este marco (GOVINDARAJAN; BAGLA, 2020).

En esa línea, India ha comenzado a profundizar los lazos con otros países en la región que están también preocupados por el ascenso chino y son aliados de EE.UU. Particularmente, India se ha unido a Japón, Australia y Estados Unidos para intensificar la actuación del Diálogo Cuadrilátero de Seguridad (Quad), una iniciativa destinada a gestionar la región Indo-Pacífico en contraposición a China (ROY-CHAUDHURI; ESTRADA, 2018).

Un cambio esencial que parece estar surgiendo es que parece cada vez más probable que Australia se sume a formar parte del próximo ejercicio naval de Malabar, que se retrasó por la pandemia Covid-19 y se celebrará a finales de 2020. Esos simulacros surgieron en los 90 entre India y Estados Unidos, y en el Siglo XXI sumaron a Japón. Hasta ahora los intentos estadounidenses por la incorporación de Australia fueron rechazados por la India. Por eso, la aceptación de la India a la participación australiana es vista por Guangyu (2020), de la Asociación China para el Control de Armas y

\footnotetext{
${ }^{7}$ Según Orchard (2018b, n.p.): “La modernización naval de la India está considerablemente por detrás de las capacidades marítimas de rápido desarrollo de China. La India está empezando a invertir fuertemente en activos de proyección de energía marítima, incluyendo una nueva flota de submarinos nucleares y sus primeros aviones de construcción local, un creciente arsenal de misiles anti buque, aviones de vigilancia marítima y aviones de guerra antisubmarinos”.

${ }^{8}$ Narendra Modi en un viaje a Houston en 2019 fue efusivamente recibido por el presidente Donald Trump. Según Shapiro (2020, n.p.): “Estados Unidos ha hecho todo lo posible para no criticar a la India por su reciente paso a abrir la autonomía política de Cachemira, un marcado contraste con la denuncia bipartidista de Washington de la relativamente práctica de China las violentas protestas en Hong Kong. Estados Unidos parece dispuesto a abandonar incluso el pretexto de una relación de cooperación con su socio de larga data Pakistán para cortejar a la India a su lado”.
} 
Desarmamento, como un movimiento que está dirigido a China por causa del reciente enfrentamiento fronterizo. También destaca que la participación de Australia agruparía por primera vez a todos los miembros del Quad, que de foro estratégico informal adquiriría una dimensión militar (GUANGYU, 2020). La India ha estado igualmente ocupada en el patio trasero de China, profundizando la defensa y la cooperación económica con los Estados que plantean problemas estratégicos a Beijing, como Vietnam, Filipinas y Singapur. Lo más alarmante para Beijing, además de los problemas políticos y económicos regionales, es la mayor disposición de la India en exportar sistemas de armas críticas, como el misil BrahMos.

Sin embargo, el movimiento indio de relacionarse con Estados Unidos para balancear el poder chino encuentra límites en la inseguridad que EE.UU. le genera como aliado. Económicamente, EE.UU. no le ha dado señales de que ocupará el lugar hoy ocupado por el comercio e inversión china. Por otro lado, también debe contemplar los efectos de un acercamiento con Washington en sus relaciones con Rusia, su principal proveedor de armas, y con Irán, uno de sus principales socios en Medio Oriente y considerado su única puerta de entrada al Asia Central (SPINDLE; ROY, 2018). Es decir, al aproximarse a EE.UU., India también acentúa varios dilemas en cuestiones claves, como la modernización de sus fuerzas armadas y el acceso a recursos energéticos críticos para el desarrollo nacional.

Además, Washington viene incrementando el embate con China en una intención que le puede generar mayores inseguridades. EE.UU. en julio de 2020, al cumplirse el cuarto aniversario de la decisión arbitral en el Mar de Sur de China, emitió una declaración alegando que la mayoría de los reclamos de Beijing en el mar son "completamente ilegales", y acusándola de intimidar a los estados costeros del sudeste asiático de recursos offshore en un intento de establecer un dominio unilateral (POMPEO, 2020). Esa declaración enérgica es una manifestación típica del endurecimiento creciente hacia China que este año ha caracterizado al gobierno de Estados Unidos.

Esto incluye también movimientos militares más fuertes en la región, como, por ejemplo, el envío de tres buques de guerra acompañados por cruceros de la marina, destructores, aviones de combate y otros aviones al Mar de China Meridional. Sobre esto, el SMCP (2020) cita al contraalmirante de EE.UU. Stephen Koehler, director de operaciones del Comando Indo-Pacífico, explicando que los mismos constituían símbolos fenomenales del poder naval estadounidense porque reflejan "la capacidad de estar presente de una manera fuerte es parte de la competencia". Bonnie Glaser, del Proyecto de Energía de China en el Centro de Estudios Estratégicos e Internacionales, sostuvo que "los chinos definitivamente describirán esto como un ejemplo de provocaciones estadounidenses, y como evidencia de que Estados Unidos es una fuente de inestabilidad en la región" (SCMP, 2020). Siendo parte de la región, India igualmente lo entiende así y por eso sus consecuencias le generan también preocupaciones.

Esto último es lo que más ha estado explorando, por su parte China, desde el enfrentamiento en junio. Sabiéndose la parte más fuerte económica y militarmente en la relación bilateral con India, por otro lado también ha manteniendo una postura más callada por ser consciente, al mismo tiempo, de que a partir de Estados Unidos, corre el riesgo de que se forme una alianza regional en su contra. Así, fundamentalmente por medio de sus medio de prensa semi-oficiales, se le alerta a India de los riegos que corre si sigue el camino propuesto por EE.UU. Este último país, así, continua siendo el foco principal, al menos por ahora, de sus preocupaciones.

\section{Un futuro incierto: dilemas de la rivalidad sino-india en el siglo XXI}

Las tensiones entre China e India en los últimos años parecen indicar que ya no hay lugar para la ambigüedad en sus relaciones. Por un lado, hay un aumento de presiones externas, acentuada por la estrategia de contención de Estados Unidos a China en Asia Oriental. Más importante aún, la expansión de los intereses externos y las vulnerabilidades de China e India crean dilemas a largo plazo y difíciles de resolver. Las crisis recurrentes en las relaciones fronterizas y económicas entre China e India son sólo síntomas del nuevo entorno geopolítico asiático. China, que tiempo atrás logró convencer a sus vecinos de que su expansión sería pacífica, ahora proyecta su influencia económica y asegura cada vez más 
sus intereses territoriales y marítimos por medios unilaterales. India, que se proyecta como el principal competidor de la hegemonía china en Asia, corre el riesgo de perder un socio importante para el desarrollo nacional y alejarse de los procesos de integración regional.

Además, India no puede acelerar una carrera armamentista y geopolítica en su entorno, donde tiene pocas condiciones para competir con Pequín. La mayor pesadilla de Nueva Delhi es precisamente quedar atrapada en la miríada de conflictos internos y en el subcontinente indio, impidiéndole volverse hacia los mares y expandir sus horizontes al resto del mundo. Además, Estados Unidos está atravesando un momento de confusión estratégica y declive relativo, lo que lo convierte en un socio a largo plazo poco confiable para India y otros aliados tradicionales como Japón, Corea del Sur y Filipinas. Al mismo tiempo, el surgimiento conjunto de China, India y otras potencias regionales de Asia en redes económicas interdependientes hacen imperativo buscar un modus vivendi relativamente estable y pacífico.

A pesar de su postura afirmativa, China tampoco debe arriesgarse a un conflicto abierto con la India o más vecinos, ante el riesgo de terminar provocando un movimiento de contención conjunto que se extienda de Tokio a Nueva Delhi. Así, se observa que tanto China como India no desean que sus conflictos bilaterales se transformen en escenarios "calientes", donde ambos quedarían susceptibles a guerras en dos o más frentes. Esta perspectiva de contención mutua asegurada debe direccionar los rumbos de la competencia sino-india para un juego de largo plazo, donde la suma de las disputas tácticas traerá cambios en el escenario estratégico de Asia.

Por lo tanto, el probable destino de la disputa geopolítica chino-india en la próxima década debe estar marcado por una guerra de posiciones. China apuesta por un camino de autonomía tecnológica para abordar las deficiencias en la intensificación de la guerra comercial con EE.UU., avanzando en la cooptación de socios por la ruta de la seda. India tiene dilemas más agudos en las esferas económica y militar: el papel de Estados Unidos y Japón en la modernización económica india es aún incierto y el escenario de una guerra en dos frentes contra China y Pakistán dificulta la expansión naval. Por otro lado, Nueva Delhi tiene un camino abierto para profundizar sus relaciones de seguridad en el Océano Índico y en el Sudeste Asiático, promocionándose como un proveedor de seguridad en rutas esenciales para el comercio global.

\section{Referências}

BHASKAR, C. Uday. After border clash with India, has China made a strategic miscalculation? South China Morning Post, Online, 10 jul. 2020. Disponible en: <https://scmp.com/comment/opinion/article/3092473/after-border-clash-india-has-china-made-strategic-miscalculation>.Acceso en: 20 ago. 2020

BREWSTER, David. Beyond the 'String of Pearls': is there really a Sino-Indian security dilemma in the Indian Ocean? In: DOYLE, Timothy (Ed.). Geoeconomics and Geosecurities in the Indian Ocean Region. London: Routledge, 2017.

CHAUDHURI, Pramit Pal. India-China face-off: Afterclash, experiment in engagementwill stop Hindustan Times, Online, 21 jun. 2020. Disponible en: <https://www.hindustantimes.com/india-news/india-china-face-off-after-clash-experiment-in-engagement-will-stop/story9UlRwRkJD99uMml1QMlOMM.html>. Acceso en 20 ago. 2020.

DEUSTCHE WELLE (DW). How realistic is India's 'Made in China' boycott campaign? Deutsche Welle, Online, 11 jun. 2020. Disponible en: <https:/www.dw.com/en/how-realistic-is-indias-made-in-china-boycott-campaign/a-53775373>. Acceso en: 20 ago. 2020.

FANG, Tien-Sze. Asymmetrical Threat Perceptions in India-China Relations. Oxford: Oxford University Press, 2013.

FEDIRKA, Allison. India Rising: The country is on the verge of becoming a major player on the world stage. Geopolitical Futures, Online, 26 jun. 2020. Disponible en :<https://geopoliticalfutures.com/india-rising/>. Acceso en 20 ago. 2020.

FRAVEL, M. Taylor. China's Sovereignty Obsession. Beijing's Need to Project Strength Explains the Border Clash With India. Foreign Affairs, Online, 26 jun. 2020. Disponible en: <https://www.foreignaffairs.com/articles/china/2020-06-26/chinas-sovereignty-obsession>. Acceso en: 20 ago. 2020.

GARVER, John W. China's decision forward with India in 1962. In: ROSS, R.; JOHNSTON, A. New directions to the study of Chinese foreign policy. California: Stanford University Press, 2006. Cap. 4, p. 86-130. 
GOVINDARAJAN, V.; BAGLA, G. As Covid-19 Disrupts Global Supply Chains, Will Companies Turn to India?. Harvard Business Review, Online, 25 mayo 2020. Disponible en: <https://hbr.org/2020/05/as-covid-19-disrupts-global-supply-chains-will-companies-turn-to-india>. Acceso en 20 ago. 2020.

GUANGYU, Xu. Strategic intent behind New Delhi's plan to invite Australia to join Malabar drill. Global Times, Online, 11 jun. 2020. Disponible en: $<$ https://www.globaltimes.cn/content/1194205.shtml>. Acceso en: 20 ago. 2020.

GUPTA, Shishir. The Himalayan face-off: Chinese assertion and the Indian riposte. Gurgaon: Hachette India, 2014.

HINDUSTAN TIMES (HT). Indian Navy deepens watch to check China ambitions Hindustan Times, Online, 29 jun. 2020. Disponible en: <https://www.hindustantimes.com/india-news/navy-deepens-watch-to-check-china-ambitions/story-ZfnCd24w0qR4nRY8XzRyjM.html>. Acceso en:20 ago. 2020 .

JIADONG, Zhang. Beijing-Delhi ties need better planning and restructuring. Global Times, Online, 8 jun. 2020. Disponible en: <https://www.globaltimes.cn/content/1193926.shtml>. Acceso en 20 ago. 2020.

MADAN, Tanvi. China Is Losing India. A Clash in the Himalayas Will Push New Delhi Toward Washington. Foreign Affairs, Online, 22jul2020a. Disponible en: < https://www.foreignaffairs.com/articles/asia/2020-06-22/china-losing-india >. Acceso en 20 ago. 2020.

MADAN, Tanvi. Fateful Triangle: How China Shaped U.S.-India Relations During the Cold War. Washington D.C.: Brookings Institution Press, 2020b.

MINISTRY OF EXTERNAL AFFAIRS, INDIA (MEA). Phone call between External Affairs Minister, Dr. S. Jaishankar and Foreign Minister of China, H.E. Mr. Wang Yi. MEA Press Release. New Delhi: Government of India, 17 jun. 2020. Disponible en: <https://www.mea.gov.in/pressreleases.htm?dtl/32765/Phone_call_between_External_Affairs_Minister_Dr_S_Jaishankar_and_Foreign_Minister_of_China_HE_Mr_Wang_Yi>.Acceso en 20 ago. 2020 .

MINISTRY OF EXTERNAL AFFAIRS, INDIA (MEA). Prime Minister's Keynote Address at Shangri La Dialogue (June 01, 2018). MEA Press Release. New Delhi: Government of India, 01 jun. 2018. Disponível en: <https://www.mea.gov.in/Speeches-

Statements.htm?dtl/29943/Prime+Ministers+Keynote+Address+at+Shangri+La+Dialogue+June+01+2018>. Acceso en: 20 ago. 2020.

MINISTRY OF FINANCE, INDIA (MF). Restrictions on Public Procurement from certain countries (July23, 2020). MF Press Release. New Delhi: Government of India, 23 jun. 2020. Disponible en: <https://pib.gov.in/PressReleasePage.aspx?PRID=1640778>. Acceso en:20 ago. 2020.

ORCHARD, Philip. China, India and the Confrontation Neither Side Wants. Geopolitical Futures, Online, 27 abril 2018 a. Disponible en: <https://geopoliticalfutures.com/china-india-confrontation-neither-side-wants/>. Acceso en 20 ago. 2020.

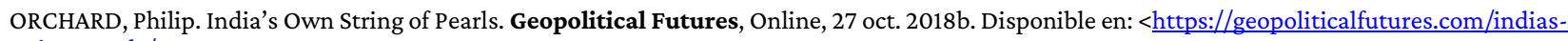
string-pearls/>. Acceso en 20 ago. 2020

POMPEO, Michael R. U.S. Position on Maritime Claims in the South China Sea. US Department of State Press Release. Washington D.C.: Department of State, 13 jun. 2020. Disponible en: <https://www.state.gov/u-s-position-on-maritime-claims-in-the-south-china-sea/>. Acceso en 20 ago. 2020.

PRESS TRUST OF INDIA (PTI). CAIT launches 'China Quit India' campaign to boycott Chinese goods. The Times of India, Online, 09 ago. 2020a Disponible en: < https://economictimes.indiatimes.com/news/politics-and-nation/cait-launches-china-quit-india-campaign-to-boycott-chinesegoods/articleshow/77446374.cms>. Acceso en 20 ago. 2020.

PRESS TRUST OF INDIA (PTI). Government imposes restriction on public procurement from China, other neighbors. The Times of India, Online, 23 jun. 2020b. Disponible en: < http://timesofindia.indiatimes.com/articleshow/77135519.cms $>$. Acceso en 20 ago. 2020.

RAJA MOHAN, C. Beyond Non-alignment: S Jaishankar's Reflections on Indian Foreign Policy. ISAS Insights, no. 589. Singapore: Institute of South Asian Studies, 2019.

RIBEIRO, Erik H. A Grande Estratégia da Índia: Ascensão de uma nova Grande Potência no século XXI? Tese (Doutorado em Estudos Estratégicos Internacionais) - Programa de Pós-Graduação em Estudos Estratégicos Internacionais. Faculdade de Ciências Econômicas, Universidade Federal do Rio Grande do Sul, Porto Alegre, 2019. 639p.

ROY-CHAUDHURI, R.; ESTRADA, K. S. India, the Indo-Pacific and the Quad. Survival, v. 60, n. 3, 2018, p. 181-194.

SAUTER, Betina T. A procura da Índia pela estabilização regional: a aproximação com a União Soviética até a Guerra de Bangladesh. Dissertação (Mestrado em Estudos Estratégicos Internacionais) - Programa de Pós-Graduação em Estudos Estratégicos Internacionais. Faculdade de Ciências Econômicas, Universidade Federal do Rio Grande do Sul, Porto Alegre, 2020. 116p. En publicación.

SOUTH CHINA MORNING POST (SCMP). US navy patrols Indo-Pacific for first time in three years, as US-China tensions deep en. South China Morning Post, Online, 12 jun. 2020. Disponible en: < https://scmp.com/news/asia/diplomacy/article/3088776/us-navy-patrols-indo-pacific-firsttime-three-years-us-china>. Acceso en 20 ago. 2020.

SHAPIRO, Jacob L. India's Fate Is in Its Own Hands. Geopolitical Futures, Online, 27 sep. 2019. Disponible en: < https://geopoliticalfutures.com/indiasfate-is-in-its-own-hands/>. Acceso en 20 ago. 2020.

SHIRK, Susan L. One-sided rivalry: China's perceptions and policies toward India. In: FRANKEL, F. R.; HARDING, H. The India-China relationship: what the United States needs to know. New York: Columbia University Press, 2004

SINGH, Zorawar Daulet. Power and Diplomacy: India's Foreign Policies During the Cold War. New Delhi: Oxford University Press, 2019. 
SPINDLE, B.; ROY, R. Iran, Russia Sanctions Vex U.S.-India Relations. The Wall Street Journal, Online, 19 jun. 2018. Disponible en: <https://www.wsj.com/articles/iran-russia-sanctions-vex-u-s-india-relations-1532001600>. Acceso en: 20 ago. 2020.

SPYKMAN, Nicholas J. The Geography of the Peace. New York: Harcourt, Brace and Co, 1944.

TELLIS, A. J.; MIRSKI, S. Crux of Asia: China, India, and the Emerging Global Order. Washington D.C.: Carnegie Endowment for International Peace, 2013.

YENDAMURI, P.; INGIZILIAN, Z. In 2020 Asia will have the world's largest GDP. Here's what that means. World Economic Forum, Online, 20 dec 2019. Disponible en: <https://www.weforum.org/agenda/2019/12/asia-economic-growth/>. Acceso en 20 ago. 2020.

Funciones de colaboración ejercidas

Andres Ferrari Haines

Erik Herejk Ribeiro
Conceituação; Metodologia; Validação; Curadoria de dados; Administração do projeto; Visualização; Investigação; Escrita (primeira redação); Escrita (revisão e edição);

Conceituação; Metodologia; Validação; Curadoria de dados; Administração do projeto; Visualização; Investigação; Escrito (primeira redação); Escrita (revisão e edição);

Información proporcionada por los(as) autores(as) según la Taxonomía de Funciones de Colaboración (CRediT) 